\title{
Manuela Massa: Sprache, Ethik und Leben bei Heidegger und Wittgenstein, Freiburg / München 2019: Karl Alber Verlag, 284 Seiten, ISBN: 978-3495490792.
}

\author{
Jan Kerkmann
}

Angenommen: 4. März 2021 / Online publiziert: 17. März 2021

(C) Der/die Autor(en) 2021

Es ist kaum zu bestreiten, dass die Konstellation ,Wittgenstein und Heidegger ' in den letzten Jahrzehnten verstärkt in den Fokus einer philosophiegeschichtlichen Selbstverortung der Moderne gerückt ist. Dabei ist es ein eminentes Verdienst der einschlägigen Forschungen, die scheinbare Dichotomie zwischen dem in der Tradition des logischen Atomismus stehenden Wiener Sprachphilosophen auf der einen Seite und dem durch die phänomenologische Schule Husserls gegangenen Seinsdenker auf der anderen Seite überzeugend hinterfragt zu haben.

In den etablierten Standardwerken von Thomas Rentsch ${ }^{1}$, Simone Venezia ${ }^{2}$ und Matthias Flatscher ${ }^{3}$ dominiert jedoch eine Vergleichsperspektive, welche die in Sein und Zeit entfaltete, relationale Ontologie pragmatischer Verweisungszusammenhänge auf die Gebrauchstheorie der Bedeutung in Wittgensteins Spätwerk bezieht. Demgegenüber begrenzt Manuela Massa den zu interpretierenden Textcorpus in ihrer 2019 im Alber Verlag erschienenen Monographie auf das Frühwerk der beiden Denker. In concreto bedeutet dies, dass im Falle Wittgensteins neben den Tagebüchern der Weltkriegsjahre ausschließlich der Tractatus logico-philosophicus beleuchtet wird. In der Auseinandersetzung mit Heidegger fließt die philosophische Entwicklung bis hin zum Hauptwerk Sein und Zeit (1927) in die Parallelisierung

\footnotetext{
1 Vgl. Thomas Rentsch, Heidegger und Wittgenstein. Existential- und Sprachanalysen zu den Grundlagen philosophischer Anthropologie, Stuttgart 1985.

2 Vgl. Simona Venezia, La misura della finitezza. Evento e linguaggio in Heidegger e Wittgenstein, Milano 2013.

3 Vgl. Matthias Flatscher, Logos und Lethe: Zur phänomenologischen Sprachauffassung im Spätwerk von Heidegger und Wittgenstein, Freiburg / München 2011.
}

J. Kerkmann ( $\square)$

Philosophisches Seminar, Albert-Ludwigs-Universität Freiburg, Freiburg, Deutschland

E-Mail: jan.kerkmann@philosophie.uni-freiburg.de 
ein. Bereits an dieser Stelle kann die sehr fruchtbringende Entscheidung der Verf. hervorgehoben werden, Heideggers frühe Freiburger Vorlesungen (1919-1923) und die intensive Aristoteles-Interpretation im Hinblick auf ihre urteilstheoretischen, semantischen und hermeneutischen Potenziale zu erschließen.

Der Titelwahl korrespondierend, lässt sich die Vergleichsanordnung der Arbeit in die drei übergeordneten Komplexe Sprache, Leben und Ethik unterteilen. Nach der Einleitung widmet sich die Autorin zunächst der nicht nur in Wittgensteins Logischphilosophischer Abhandlung, sondern auch in Heideggers Frühphilosophie thematisierten Fragestellung nach den Grenzen sprachlicher Aussagbarkeit. Für Wittgenstein lassen sich die Sätze als aus gegenstandsbezogenen Namen zusammengesetzte Kommunikationseinheiten verstehen, die bestehende Sachverhalte abbilden. Da die Sprache als Gesamtheit aller überhaupt in Sätzen fassbaren Gedanken ${ }^{4}$ definiert werden kann und die nach formalen logischen Eigenschaften konstruierten, sinnvollen Sätze wiederum auf die festen Formen der Gegenstände rekurrieren müssen, ist es prinzipiell unmöglich, sich die Welt in einer metasprachlichen Ebene begegnen zu lassen. Daraus folgert Wittgenstein bekanntlich, dass die „Grenzen der Sprache [...] die Grenzen meiner Welt" 5 bedeuten. Wie Massa aufzeigt, lässt sich für den Heidegger der 1920er-Jahre eine analoge Unhintergehbarkeit der sprachlichen Vermittlung behaupten, insofern das Existenzial der Rede jene Verständlichkeit gliedert, durch die der entwerfende Vollzugssinn des Daseins allererst die - in dem jeweiligen Worumwillen fundierten - Verweisungszusammenhänge aufzuspannen vermag. ${ }^{6}$ Obwohl Massa in einem generellen Sinne unterstreicht, dass die „Hermeneutik bei Heidegger den Sinn des Seins betrifft“ und ,,bei Wittgenstein auf Sprache und Logik bezogen“" (64) ist, kann kritisch angemerkt werden, dass die beiden Denker in ihren hermeneutischen Grundauffassungen zu wenig voneinander abgegrenzt werden. So lässt sich Wittgensteins Impetus, dass sich die ganze Welt vollständig durch allgemeine Sätze beschreiben ließe, die sich aus unabhängigen Elementarsätzen aufbauen ${ }^{7}$, prima facie kaum mit Heideggers Herausarbeitung eines vorprädikativen Verstehens versöhnen, aus dem die Modi der Auslegung - Aufzeigung, Mitteilung und Prädikation ${ }^{8}$ - und damit die in Sätzen artikulierte Aussagenwahrheit erwachsen sollen.

\footnotetext{
${ }^{4}$ Vgl. Ludwig Wittgenstein, Tractatus logico-philosophicus [im Folgenden = TLP], 3.032: „Etwas ,der Logik widersprechendes ' in der Sprache darstellen, kann man ebensowenig, wie in der Geometrie eine den Gesetzen des Raumes widerstreitende Figur durch ihre Koordinaten darstellen; oder die Koordinaten eines Punktes angeben, welcher nicht existiert.“.

5 Wittgenstein, TLP, 5.62 .

${ }^{6}$ Vgl. Martin Heidegger, Sein und Zeit, §32, Tübingen 2006, S. 149: „Alles vorprädikative schlichte Sehen des Zuhandenen ist an ihm selbst schon verstehend-auslegend. [...] Es birgt in sich die Ausdrücklichkeit der Verweisungsbezüge (des Um-zu), die zur Bewandtnisganzheit gehören, aus der her das schlicht Begegnende verstanden ist. Die Artikulation des Verstandenen in der auslegenden Näherung des Seienden am Leitfaden des ,Etwas als Etwas' liegt vor der thematischen Aussage darüber.“.

${ }^{7}$ Vgl. Wittgenstein, TLP, 5.526.

8 Vgl. Heidegger, Sein und Zeit, §34, S. 161: „Rede ist die Artikulation der Verständlichkeit. Sie liegt daher der Auslegung und Aussage schon zugrunde. Das in der Auslegung, ursprünglicher mithin schon in der Rede Artikulierbare nannten wir den Sinn.“.
} 
Heideggers vorbegriffliche Struktur des hermeneutischen Als hätte allerdings gemeinsam mit Wittgensteins transzendentalphilosophischer Überlegung verhandelt werden können, wonach der Satz durch interne Eigenschaften und logische Formen gebildet wird, die von ihm nicht selbstreferentiell ausgesagt, sondern als Bedingungen seiner eigenen Möglichkeit nur in ihm aufgewiesen werden können. ${ }^{9}$ Erst durch den Gebrauch des Namens in einem syntaktischen Ordnungsgefüge lässt sich den Gegenständen ihre vollständige Form prädizieren, woraus sich ergibt, dass es ohne Sprache ,unmöglich [wäre], zu einer Wirklichkeit oder zu Sachverhalten zu gelangen“ (90f.). Darauf aufbauend, hätte der fundamentallogische Kerngedanke, dass die „Sprache für Wittgenstein außerhalb der Logik unmöglich“ ist (67), mit Heideggers Deutung des Logos als Entdeckungsgarant des Sich-Zeigens der Unverborgenheit des Seienden in ein Verhältnis gesetzt werden können. In diesem Kontext hätte trennscharf bestimmt werden können, in welcher Weise das genuine Sehenlassen des Logos nach Heidegger sprachlich strukturiert ist.

Da Heidegger die Logik in seiner Marburger Vorlesung zum Sophistes ausdrücklich auf der Onto-logie fußen lässt (vgl. 77) und Wittgensteins Tractatus demonstrativ mit den Entitäten der Gegenstände, Tatsachen, Sachverhalte, Eigenschaften und Strukturen operiert, ist Massas Motivation nachvollziehbar, die Ontologien beider Denker im 3. Kapitel (79-112) vergleichend aufeinander zu beziehen. Hier beruft sich die Verf. zunächst auf Wittgensteins Tagebuchnotiz vom 16.06.1915. Gemäß dieser Aufzeichnung sind auch die Relationen und Eigenschaften als Gegenstände zu benennen (vgl. 90). ${ }^{10}$ Wenn der Satz allein den bestehenden Sachverhalt abbildet, kann er den innerontologischen Status eines Gegenstandes nicht angeben. Das Sein des Satzes bezeugt sich in dem Ausdruck des „Wie“ der Koexistenz eines Dinges mit anderen Tatsachen. Die Ontologie bleibt demnach ,für Wittgenstein weiterhin in der Logik verankert“" (91), da sich die Essenz des Gegenstandes nicht durch die Aufzählung materieller Eigenschaften gewinnen lässt. Vielmehr ist es das potenzielle Vorkommnis des Gegenstandes in spezifischen Sachverhalten des logischen Raumes, das dem Ding als dessen Präjudiz inhäriert. Die sachgerechte Erschließung des Dinges fällt folglich mit der umfassenden Kenntnis aller seiner internen Eigenschaften zusammen. ${ }^{11}$ Auch für Heidegger wird die seinsmäßige Bedeutsamkeit eines Dinges nicht durch primäre Qualitäten innerhalb der res extensa konturiert. In Übereinstimmung mit der epistemologischen Zentralaussage des Tractatus $^{12}$ bezweifelt Heidegger außerdem die ontologische Erstrangigkeit des vorstellenden und denkenden Subjektes der cartesischen Tradition.

Im Kontrast zu Wittgenstein gründet diese Depotenzierung des Subjekts bei Heidegger nicht darin, dass das logische Subjekt als bedingende Grenze der Welt fungiert. Stattdessen zielt Heideggers Ontologie darauf ab, das Sein des von Descartes als Zweifelsresultat befestigten Sum zu erfragen. Das Subjekt wird in Heideggers früher Hermeneutik der Faktizität also nicht als ichhaftes Substrat weltstrukturierender Kategorien diskutiert. Vielmehr soll die alltägliche Selbstauslegung des konkret-

\footnotetext{
9 Vgl. Wittgenstein, TLP, 4.122 .

10 Vgl. Wittgenstein, Tagebücher [im Folgenden = TB], 16.06.1915.

11 Vgl. Wittgenstein, TLP, 2.01231.

12 Vgl. Wittgenstein, TLP, 5.631.
} 
geschichtlichen Individuums in den Blick geraten, das sich immer schon in einer bedeutungstragenden, durch das Mitsein mit anderen Menschen geprägten Welt vorfindet. Dergestalt vermeidet Heidegger bereits in der ursprünglichen Anlage seiner Konzeption jenen Solipsismus, dessen Wahrheitsanspruch Wittgenstein im Tractatus nicht zu leugnen vermag. ${ }^{13}$ Es wäre wünschenswert gewesen, wenn die Verf. diese gravierende Divergenz zwischen Heidegger und Wittgenstein im 4. Kapitel (Das Vortheoretische bei Heidegger und Wittgenstein) deutlicher markiert hätte. So ist es fraglich, ob Wittgenstein die Annahme einer nicht-psychologischen, transzendentalen Subjektivität, die in Heideggers existenzialer Rekonstruktion der Metaphysik immer schon in die Intentionalität des jeweiligen Besorgens eingebunden ist und daher nur in einer abstrahierenden Ausdünnung zur weltbegrenzenden Konstitutionsinstanz hypostasiert werden kann - wirklich kritisieren möchte. Die Verf. scheint dies selbst einzuräumen, wenn sie schreibt, dass „dem Subjekt die Welt ,gehört“", „insofern sie unter einer logischen Ordnung steht“ (179).

Im Tractatus wird das Subjekt stets im Singular genannt. Diametral dazu, kann es in Sein und Zeit kein Dasein geben, dass sich aus den Kollektivstrukturen des Man, des Mitseins, und aus den durch die Überlieferungstradition vorgezeichneten Möglichkeiten endgültig herauslösen könnte. Die grundlegende Hürde für eine systematische Bedeutungsuntersuchung der Intersubjektivität äußert sich also darin, dass das In-der-Welt-sein bei Heidegger als unabweisliches Existenzial des Daseins betrachtet werden muss, wohingegen der frühe Wittgenstein die Beurteilung der innerweltlichen Situierung des Menschen der empirischen Psychologie zuweisen würde. Mit Kant insistiert Wittgenstein auf der Unmöglichkeit einer materialen Selbsterkenntnis des Subjekts. Vor diesem Hintergrund ist es als großes Verdienst der Studie zu würdigen, dass Massa sich eingehend mit der scheinbaren Unvereinbarkeit der phänomenologischen Lebensweltanalyse Heideggers und der logischen Formaldeutung Wittgensteins befasst. Der die Kapitel Nr. 7, 8 und 9 beinhaltende Abschnitt zur Ethik und Normativität kann mit gutem Recht als philosophisch aussagekräftigster und inhaltlich ergiebigster Textbereich der vorliegenden Monographie bewertet werden.

Es ist ersichtlich, dass weder Heidegger noch Wittgenstein eine präskriptive Ethik vertreten, die sich auf die Begriffe einer sittlichen Pflicht oder eines absolut Guten berufen könnte und den Wertkategorien von ,Gut und Böse" eine objektiv-überzeitliche Existenz zuspricht. Ebenso wenig sind beide Denker an einer psychologischen Ergründung der moralischen Triebfedern interessiert. Um dennoch den tiefen ,ethischen Sinn" (188) im Frühwerk beider Denker hervorheben zu können, favorisiert Massa einen phänomenologisch-normativen Zugang (vgl. 196-219). Dieser wird zunächst auf Heideggers existenzial-säkularisierte Interpretation des Gewissensrufes angewendet, der - indem er im Rufverstehen das ursprüngliche Schuldigsein des Daseins zum Ausdruck bringt und dieses zu seinem eigentlichen Selbstsein auffordert genuin ethische Motive besitzt. Anhand des Topos der ,Eigentlichkeit ' des Daseins sucht Massa die „formalen Bedingungen des Ethischen“ (196) bei Heidegger zu illustrieren. Es fügt sich harmonisch in diesen Ansatz ein, dass die Entschlossenheit

13 Vgl. Wittgenstein, TLP, 5.641: „Das Ich tritt in die Philosophie dadurch ein, daß die ,Welt meine Welt ist". ". 
nicht als heroische Emanzipation von den Entfremdungsangeboten des Man gefasst wird. Umsichtig interpretiert Massa die Entschlossenheit als ,moralische Offenheit“, die eine freie Neuauslotung des ,durch das Verstehen gegebenen Horizont des Mitseins“ (199) verspricht. Die im Rufcharakter des Gewissens hinterlegte Entdeckung des eigenen Möglichseins bedeutet also, sich in der unweigerlich mit Anderen geteilten Welt nicht mehr durch gesellschaftlich geforderte Entscheidungsraster determinieren zu lassen. Umgekehrt gilt es, im Zuge der Selbstwahl den Modus des Mit- und Zueinanderseins in einer zentrifugalen Bewegtheit eigenständig zu gestalten. Dergestalt lässt erst die in der Entschlossenheit verwurzelte Verantwortlichkeit für die innerweltliche Tragweite der eigenen Aktivität eine wahrhafte Interaktion zwischen ,Ich“ und ,Du“ zu.

Heidegger bekräftigt durch das in Sein und Zeit aufgerufene, pathetische Begriffsfeld - Schuld, Gewissen, Selbstwahl, Mitsein, Fürsorge, Tod - dass die existenziale Analyse der Alltäglichkeit durch eine dezidiert ethische Schicht fundiert wird. Wie Massa zu Beginn ihres „,Vorgriff[es] auf eine Handlungstheorie und Willensreglementierung bei Wittgenstein“ (203) treffend expliziert, lässt sich in der nüchternen Terminologie des Tractatus auf den ersten Blick kein vergleichbares, ethisches Gravitationszentrum auffinden. Zum ersten bezweifelt Wittgenstein die für jede Handlungstheorie konstitutive Beeinflussungsoption der Welt durch den Willen, wenn er lapidar festhält: „Die Welt ist unabhängig von meinem Willen“. ${ }^{14}$ Zum zweiten scheint Wittgenstein jeder moralisch-qualitativen Differenzierung das Fundament zu entziehen, wenn er mit der These aufwartet, dass Gut und Böse ,wesentlich nur das Ich, und nicht die Welt" ${ }^{\prime 15}$ (212) seien. Dementsprechend vermag das gute und böse Wollen auch nicht auf die sprachlich auszudrückenden Tatsachen innerhalb der Welt einzuwirken; es kann allein die Grenzen der Welt ändern, an denen das Ich positioniert ist. ${ }^{16}$ Dass Massa den Willen vollkommen zurecht als „Instanz des Ethischen“ (203) in Wittgensteins Denken benennt, wird spätestens dann deutlich, wenn Wittgenstein die Transformation des Wollens - trotz dessen Ohnmacht hinsichtlich der Geschehnisse der Welt - mit einer tiefgreifenden ethischen Zäsur zusammenfallen lässt, die sich in dem berühmten Satz verdichtet: „Die Welt des Glücklichen ist eine andere als die des Unglücklichen." 17

Auch wenn Massa den theologischen Aspekten im Denken Wittgensteins keinen allzu großen Raum schenkt, legt sie im Kapitel 7.3. eine wichtige Spur zu der religiös-soteriologischen Sinnsuche des jungen Philosophen frei. Zentral ist dabei die schon 1916 im Tagebuch aufgezeichnete Reflexion Wittgensteins, wonach die „Welt mir gegeben [ist] d. h. mein Wille tritt an die Welt ganz von außen als an etwas Fertiges heran“18 (vgl. 209). Massa zieht daraus die triftige Schlussfolgerung, dass die Existenz der Welt keinen Willen voraussetze. Dies impliziert jedoch auch gegen den Anschein eines teilnahmslos-passiven Fatalismus -, dass die Bewegungsrichtung, aus welcher der faktische, die Handlungen des Subjekts begleitende Wille

\footnotetext{
14 Wittgenstein, TLP, 6.373.

15 Wittgenstein, TB, 5.8.16.

16 Vgl. Wittgenstein, TLP, 6.43.

17 Wittgenstein, TLP, 6.43.

18 Wittgenstein, TB, 8.7.16.
} 
ursprünglich an die Welt herantritt, nicht von den Objekten innerhalb des Konnexes der Sachverhalte limitiert wird. Der Wille zielt überschreitend auf ein Etwas hin, das „sich jenseits der Welt befindet“ (209).

Im letzten Kapitel (251-271) der Monographie wird mit dem Tod ein Thema in den Mittelpunkt gerückt, das in den bisherigen Arbeiten zur Konstellation ,Heidegger und Wittgenstein ' kaum als gemeinsamer Referenzpunkt gewürdigt wurde. Dies muss insofern überraschen, als die zumeist mit dem Denken Heideggers verbundene Umkreisung der Demarkationslinie menschlicher Zeitlichkeit in den - unter der Kennziffer Nr. 6 versammelten - Überlegungen des Tractatus mit einer herausfordernden Eindeutigkeit und Prägnanz beantwortet wird. In Heideggers Sein und Zeit soll der Entwurf auf die schlechthin gewisse, unüberholbare und äußerste Möglichkeit des Todes das eigentliche Ganzseinkönnen verbürgen. ${ }^{19}$ In der vorauslaufenden Enthüllung seiner Endlichkeit hält sich das Dasein einen Spiegel entgegen, der die für wertvoll erachteten, sinnstiftenden Zielsetzungen einfängt und zu einer Ernsthaftigkeit der Lebensführung verpflichtet. Gerade im Vorblick auf eine markante Abgrenzung gegenüber der Thanatologie Wittgensteins hätte in diesem Kontext noch ausdrücklicher apostrophiert werden sollen, dass das Sein zum Tode für Heidegger unmittelbar in den Lebensvollzug zurückschlägt, aus der „Unpersönlichkeit des Massendaseins“ (266) befreit und sich als permanenter Begleiter des Einzelnen enthüllt.

Im Grunde ist nach Heidegger jedes Sich-verhalten des Daseins zur Welt - sei es im Modus des verfallenden Besorgens, sei es im Untertauchen in der Anonymität des Man oder in der wiederholenden, lebensfördernden Erschließung geschichtlicher Vorbilder - durch die unbewusst verdrängte oder desillusioniert akzeptierte Unumgänglichkeit des Todes geprägt. Der Tod darf nach Heidegger - wie Massa anschaulich nachzeichnet - also nicht als ein unerfahrbares und daher belangloses Faktum in einem unbestimmbaren Niemandsland verortet werden. Insofern Heidegger die subtilen Verdrängungsmuster des Sterbenmüssens dekuvriert, hätte der Verdacht formuliert werden können, ob nicht ebendiese Verharmlosung des Todes in Wittgensteins Tractatus zu philosophischen Weihen aufsteigt. So ist kaum zu leugnen, dass Wittgenstein sich nicht nur in der Einzeichnung eines Hiatus zwischen Leben und Tod (,,der Tod ist kein Ereignis des Lebens. Den Tod erlebt man nicht. “" von Heidegger abhebt. Auch Wittgensteins Verkopplung des ewigkeitserfüllten Lebens mit der Prävalenz präsentischen Existierens ${ }^{21}$ dürfte wohl kaum Heideggers Zustimmung gefunden haben. Schließlich ist es seit den frühen 1920er Jahren das erklärte Anliegen Heideggers, die ,Bewegtheitscharaktere des faktischen Lebens“ in ihrer radikalen Endlichkeit zu erkunden und auf den für das Seinsverständnis unverzichtbaren Horizont der Zeit aufmerksam zu machen. Leider dominiert auch hier Massas generelle Interpretationsstrategie einer Überbewertung der Gemeinsamkeiten bei gleichzeitiger Einebnung der unübersehbaren Gegensätze. Dergestalt gelangt sie

\footnotetext{
19 Vgl. Heidegger, Sein und Zeit, §53, S. 264.

20 Wittgenstein, TLP, 6.4311.

21 Vgl. Wittgenstein, TLP, 6.4311: ,Wenn man unter Ewigkeit nicht unendliche Zeitdauer, sondern Unzeitlichkeit versteht, dann lebt der ewig, der in der Gegenwart lebt. Unser Leben ist ebenso endlos, wie unser Gesichtsfeld grenzenlos ist.“.
} 
zu dem etwas unterkomplex wirkenden Ergebnis, dass Heidegger und Wittgenstein ein glückliches Leben in der Gegenwart als Existenzideal präferierten. Auf diese Weise wird allerdings der Primat der Zukunftsekstase in Sein und Zeit verkannt.

Ein rein an der Gegenwart orientiertes Leben müsste für Heidegger in einer problematischen Nähe zu der in der Alltäglichkeit vorherrschenden, verfallenden Selbstabriegelung gegenstandsbezogener Welthandhabe angesiedelt werden. Des Weiteren könnte erstens bezweifelt werden, ob der Tod im Tractatus tatsächlich als Kriterium betrachtet werden darf, das ,,die Welt definiert, die innerhalb unserer Erfahrung hervorgebracht wird“ (269). Gegen diese vonseiten Massas proponierte Relevanz des Todes im Tractatus spricht, dass Wittgenstein im selben Werk auf der - den Tod schlechthin exkludierenden - Identität von Leben und Welt ${ }^{22}$ beharrt, weswegen das Ich einen abgeschlossenen ,Mikrokosmos“23 auszufüllen vermag. In diesem Zusammenhang kann zweitens die Rückfrage adressiert werden, ob Wittgenstein das als glücklich gekennzeichnete, weder von Furcht noch von Hoffnung tangierte Leben mit einem Verzicht auf den „Eingriff in die Ereignisse, die in der Welt geschehen“ (271) assoziiert. Drittens ließe sich problematisieren, wie sich die als Seismogramm des Glücklichseins bestimmte, genügsame Übereinstimmung mit der Welt zu der am Ende der Monographie aufgegriffenen Transzendenzrichtung ${ }^{24}$ der Eudämonologie Wittgensteins verhält. Die Lösung der Sinnfrage verweist nach Wittgenstein auf eine „Verbindung zu Gott“ (271), welche die proklamierte Gegenwartskonzentration notwendigerweise irritieren oder sogar erschüttern müsste.

Gerade die Betonung der diametral entgegengesetzten Positionierungen zum Thema des Todes hätte ein fruchtbares Zwiegespräch zwischen Wittgenstein und Heidegger eröffnen können. In diesem Diskurs hätten zwei gleichsam archetypische Betrachtungsweisen des unauslöschlichen Rätsels der Endlichkeit zum Vorschein kommen können. Trotz der oben genannten Kritikpunkte soll die Qualität von Massas wichtiger und aufschlussreicher Studie keineswegs geschmälert werden. Aufbauend auf einer profunden und heterogene Themenbereiche umfassenden Kenntnis des Frühwerkes beider Denker arbeitet die Autorin in aller wünschenswerten Deutlichkeit heraus, dass sowohl Wittgensteins als auch Heideggers frühes Denken in einem bislang ungeahnten Maße von einem ethischen Impuls durchdrungen ist. Massas Untersuchung erbringt den überzeugenden Nachweis, dass sich die Abgründigkeit des Ethischen sowohl in der logisch-semantischen Grenzbestimmung der Weltbedeutsamkeit als auch in Wittgensteins und Heideggers Ringen um eine neue Philosophie erkennen lässt. Dieses gehaltvolle Forschungsergebnis wird der künftigen Diskussion sicherlich neue Perspektiven erschließen.

Funding Open Access funding enabled and organized by Projekt DEAL.

\footnotetext{
22 Vgl. Wittgenstein, TLP, 5.621: „Die Welt und das Leben sind Eins.“.

23 Wittgenstein, TLP, 5.63: „Ich bin meine Welt. (Der Mikrokosmos).“.

24 Vgl. Wittgenstein, TLP, 6. 41: „Wenn es einen Wert gibt, der Wert hat, so muß er außerhalb alles Geschehens und So-Seins liegen. Denn alles Geschehen und So-Sein ist zufällig. Was es nicht-zufällig macht, kann nicht in der Welt liegen, denn sonst wäre dies wieder zufällig. Es muß außerhalb der Welt liegen.".
} 
Open Access Dieser Artikel wird unter der Creative Commons Namensnennung 4.0 International Lizenz veröffentlicht, welche die Nutzung, Vervielfältigung, Bearbeitung, Verbreitung und Wiedergabe in jeglichem Medium und Format erlaubt, sofern Sie den/die ursprünglichen Autor(en) und die Quelle ordnungsgemäß nennen, einen Link zur Creative Commons Lizenz beifügen und angeben, ob Änderungen vorgenommen wurden.

Die in diesem Artikel enthaltenen Bilder und sonstiges Drittmaterial unterliegen ebenfalls der genannten Creative Commons Lizenz, sofern sich aus der Abbildungslegende nichts anderes ergibt. Sofern das betreffende Material nicht unter der genannten Creative Commons Lizenz steht und die betreffende Handlung nicht nach gesetzlichen Vorschriften erlaubt ist, ist für die oben aufgeführten Weiterverwendungen des Materials die Einwilligung des jeweiligen Rechteinhabers einzuholen.

Weitere Details zur Lizenz entnehmen Sie bitte der Lizenzinformation auf http://creativecommons.org/ licenses/by/4.0/deed.de. 